Gefässchirurgie 2009 · 14:6-7 DOI 10.1007/s00772-008-0656-y

Online publiziert: 21. Januar 2009

๑) Springer Medizin Verlag 2009

\author{
K. Rückert · F. Kümmerle \\ Abteilung für Allgemein-, Viszeral- und Gefäßchirurgie, \\ Asklepios Klinik Nord Heidberg, Hamburg
}

\title{
Was ist Qualität in der Chirurgie?
}

erfüllt“. Eine wesentlich konkretere Definition gab die vorherige Normfassung: Qualität wurde als Erfüllung von festgelegten und erwarteten Anforderungen beschrieben. Über die Anforderungen entscheidet in der Regel der Kunde. Die Erfüllung dieser Anforderung liegt in der Verantwortung der Hersteller von Produkten oder der Institutionen, die Dienstleistungen erbringen.

\section{Welche Qualitätsgesichts- punkte könnten für die Medizin, in engerem Sinne für die Chirurgie gelten?} lität im Handel: „Beschaffenheit einer Ware nach ihren Unterscheidungsmerkmalen gegenüber anderen Waren, ihren Vorzügen oder Mängeln“. Man unterscheidet objektive Kriterien: d. h. messbare Eigenschaften oder subjektive Kriterien: Abstufung des Eignungswertes gleichartiger Güter für die Befriedigung bestimmter Bedürfnisse. Der Verbraucher, hier im Sinne des Patienten, bildet Qualitätsstufen, während der Unternehmer Preisstufen bildet, d. h. Anpassung im Markt. Zum Beispiel können sich Patienten in Russland den Einsatz der minimal-invasiven Chirurgie nur gegen Vorkasse im Krankenhaus erkaufen.

Im Rahmen der externen Qualitätssicherung (EQS, BQS) wird die Qualität definiert nach der Prozess-, Struktur- und Ergebnisqualität. Sie ist der Teil des Qualitätsmanagements, der auf das Erzeugen von Vertrauen gerichtet ist, dass Qualitätsanforderungen erfüllt werden.

In der deutschen Industrienorm (DIN) ist Qualität definiert als „Grad, in dem ein Satz inhärenter Merkmale Anforderungen
1975 wurde von Vollmar, einem der ersten deutschen universitären Gefäßchirurgen in Heidelberg, das 3-S-Prinzip beschrieben, d. h. „safer“, „simpler“ und „shorter". Dies bedeutet:

- eine begrenzte, einfache Diagnostik, d. h. nicht das Aneinanderreihen von diagnostischen Methoden wie Ultraschall, Kernspintomographie und CT bis zur diagnostischen Erschöpfung des Patienten;

- eine korrekte Indikationsstellung z. B. Gallenblasenentfernung nur bei nachgewiesenen Gallenblasensteinen mit Beschwerden;

- eine möglichst ambulant oder mit der kürzestmöglichen Verweildauer durchzuführende Operation;

- eine billige und einfache Durchführung der operativen Eingriffe (z. B. keine Einmalinstrumente);

- eine „schmerzfreie“ Operation im „schmerzfreien“ Krankenhaus (man beachte die Begriffe „Gesundheitshaus“ oder „Gesundheitskasse");
- die Durchführung eines operativen Eingriffs ohne Komplikationen. Die Gefährdung liegt darin, dass eine Selektion der guten Patienten stattfindet („Rosinenpicken“).

- Die Voruntersuchungen sollen ausreichend, wirtschaftlich und angemessen sein (so die Definition im deutschen Gesundheitswesen). Im postoperativen Verlauf gilt das moderne Prinzip des „fast track“. Bei den Komplikationen ist zu unterscheiden zwischen allgemeinen und speziellen eingriffsbezogenen Komplikationen bis hin zur Letalität.

Die moderne Chirurgie soll sich an Standards orientieren (SOPs), leitliniengerecht sein, evidenzbasiert, qualitätsgesichert und wirtschaftlichkeitsorientiert. Unstrittig erscheint uns in der Diskussion, dass zu einer qualitativ hochwertigen Chirurgie ein möglichst „kompetenter Chirurg“ benötigt wird. Das Royal College of Surgeons in England definiert die Kompetenz als Summe von Fähigkeiten, die sich aus Können („skill“), Wissen („knowledge“) und psychosozialem Verhalten („attitude") zusammensetzen.

Die Persönlichkeit eines Chirurgen, seine persönliche Verantwortung und Verantwortlichkeit machen ihn zu einem kompetenten Chirurgen.

Für die erfolgreiche Behandlung eines Patienten ist die richtige Indikation von entscheidender Bedeutung: Das patientenabhängige Risiko ist möglichst objektiv und quantitativ abzuschätzen; zu bedenken ist aber auch, was dem Patienten statt ihm zu nutzen auch schaden, ihn gefährden kann. Die indikatorische Entschei- 
dung hängt von der persönlichen Einschätzung des Chirurgen ab - ob, wann und wie operiert wird -, von der persönlichen Entscheidung des Chirurgen, von seiner Erfahrung, von seinem theoretischen Wissen, aber nicht zuletzt von seiner Risikobereitschaft.

Die richtige Entscheidung im individuellen Patientenfall erfordert neben der Diagnosestellung das Berücksichtigen von Komorbidität und anderen biologischen Größen wie Alter und Allgemeinzustand des Patienten. Der rein technische Aspekt einer Operation spielt eher eine untergeordneteRolle. Somit macht die richtige Indikation und Entscheidung für jeden einzelnen, individuellen Patienten einen Chirurgen zu dem, was er ist, und spielt somit die zentrale Rolle! „Nicht die Operation, sondern der Operateur rettet den Patienten!“ (Martin Kirschner, 18791942).

Daraus ergeben sich auch wichtige Konsequenzen für die Weiterbildung:

In einem Ausbildungscurriculum müssen auch minimale Standards im Hinblick auf den Erwerb chirurgischer Kompetenz definiert werden. Das bisherige Ausbildungsprinzip, das den handwerklichen Aspekt des Chirurgen in den Vordergrund stellt, muss erweitert werden im Sinne eines Tutorprinzips, bei dem der angehende Chirurg unter Supervision lernt. Dies resultiert aus folgender Erkenntnis: Behandlungsfehler sind selten allein auf einen technischen Mangel, son- dern vielmehr auf einen Fehler in der Interpretation von Symptomen und in der Indikationsstellung zurückzuführen.

Vergessen wird häufig, dass zur Operation auch die Phase nach der Operation gehört. Die Nachbehandlung unter täglicher, unter Umständen auch mehrfacher Visite durch den Operateur ist so wichtig wie die Operation selbst.

Intuitive Entscheidungen, sozusagen aus dem Bauch heraus, sind oft schneller, ökonomischer und besser auch im ethischen Sinn.

Leitlinien bergen das Risiko in sich, das Mittelmaß zu zementieren („Kochbuchmedizin“). Das Konzept des modularen Krankenhauses (Aachen) hält den modernen Qualitätsansprüchen nicht stand und ist daher abzulehnen. „Barfußärzte“ in der Vor- und Nachbehandlung operierter Patienten erlauben keine gute Qualität.

Im Zeitalter der Ausweitung von Macht- und Kontrollräumen in der Medizin - eingeführt durch den Druck der Ökonomisierung - erschließt sich rasch das weite Feld der „Qualitätssicherung“.

Qualitätsziele, Qualitätsindikatoren, Qualitätsmessinstrumente, Qualitätssicherung und Qualitätsmanagement haben für uns immer noch etwas Abstraktes an sich, so sehr auch praktische, ökonomische, patientenindividuelle und ethische Implikationen damit verbunden sind.

Wenn es um die Ausweitung von Macht- und Kontrollräumen (= Qualitätssicherung) in der Medizin geht, kommen wir in der Gegenwart an, im Zeitalter der evidenzbasierten Medizin und der Leitlinien, die das ärztliche Handeln festlegen.

Richtlinien $=$ Standards lassen keinen Spielraum für Entscheidungen zu. Im Gegensatz zu Leitlinien (= Entscheidungskorridor), bei denen das Korsett nicht so eng geschnürt wird und noch Spielraum bleibt, fachbezogen für die individuelle Entscheidung, für die Intuition als Summe jeglicher Erfahrung.

Qualitativ hochwertige Chirurgie und wirtschaftliches Denken haben durchaus eine ethische Herausforderung, v. a. wenn im Hintergrund das heiße Eisen der Rationierung lauert. Hierzu werden wir immer den guten Arzt brauchen mit seiner Menschlichkeit, der sich aufgrund seines Wissens, Könnens und seiner Erfahrung des kranken Menschen annimmt, der ihm den richtigen Weg weist durch die parzellierte Medizin, dorthin wo ihm am besten geholfen werden kann.

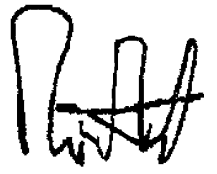

Prof. Dr. K. Rückert

\section{Korrespondenzadresse \\ Prof. Dr. K. Rückert}

Abteilung für Allgemein-, Viszeral- und Gefäßchirurgie, Asklepios Klinik Nord Heidberg Tangstedter Landstr. 400, 22417 Hamburg

k.rueckert@asklepios.com

\section{Exklusiv für Abonnenten und Gesellschaftsmitglieder :}

\section{Nutzen Sie das Online-Archiv der Zeitschrift Gefässchirurgie}

Ihre Vorteile:

- Komfortable und schnelle Recherche nach Themen, Autoren, Suchbegriffen

- Ob unterwegs oder am eigenen PC: Zugriff überall und jederzeit

— Online First: Lesen Sie die aktuellsten Beiträge schon vor Erscheinen des gedruckten Heftes online

\section{Registrieren Sie sich jetzt unter}

www.Gefaesschirurgie.springer.de

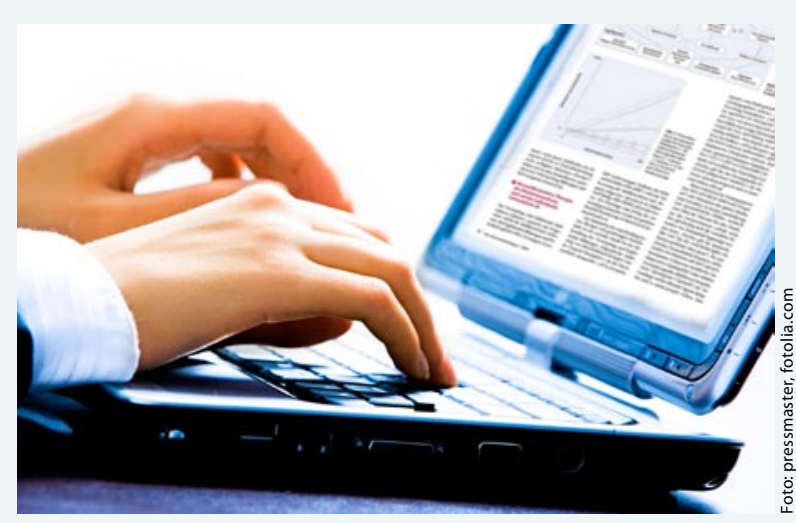

Dieser Online-Service steht auch Mitgliedern der folgenden Fachgesellschaften zur Verfügung:

- Deutsche Gesellschaft für Gefässchirurgie

- Österreichische Gesellschaft für Gefässchirurgie

- Schweizerische Gesellschaft für Gefässchirurgie 